\title{
ÉCRITURE LASER DE MATERIAUX FONCTIONNELS INORGANIQUES PREPARÉS PAR VOIE SOL-GEL
}

$/ / / / / / / / / / / / / / / / / / / / / / / / / / / / / / / / / / / / / / / / / / / / / / / / / / / / / / / / / / / / / / / / / / / / / / / / / / / / / /$

Laurent NOEL ${ }^{1,2}$, Amine KHITOUS ${ }^{1,2}$, Quentin KIRSCHER ${ }^{1,2}$,

Céline MOLINARO ${ }^{1,2}$, Dominique BERLING ${ }^{1,2}$, Olivier SOPPERA ${ }^{1,2 \star}$

${ }^{1}$ Université de Haute-Alsace, CNRS, IS2M UMR 7361, F-68100 Mulhouse, France

${ }^{2}$ Université de Strasbourg, France

*olivier.soppera@uha.fr

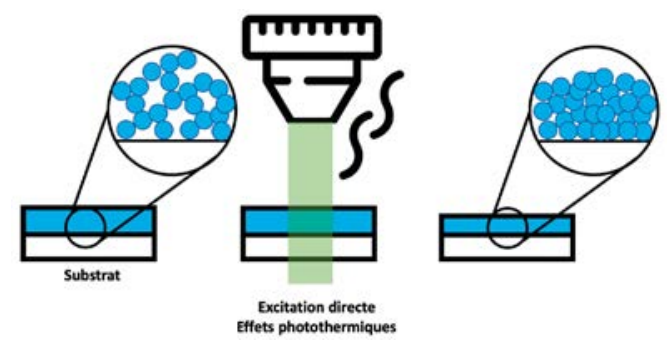

https://doi.org/10.1051/photon/202211243

ArticlepubliéenaccèslibresouslesconditionsdéfiniesparlalicenceCreative Commons Attribution License CC-BY (https://creativecommons.org/ licenses/by/4.0), qui autorise sans restrictions l'utilisation, la diffusion, et la reproduction sur quelque support que ce soit, sous réserve de citation correcte de la publication originale.

Les matériaux semi-conducteurs ont des propriétés optoélectroniques intéressantes pour de nombreuses applications microélectroniques. Leur introduction sous forme de films minces sur des substrats fragiles, tels que du verre fin, des feuilles de plastique souple ou des pièces imprimées en 3D, permet de créer de nouveaux matériaux intelligentsen introduisant des capteurs ou des photodétecteurs. Dans cet article, nous illustrons l'intérêt denouvellesapproches baséessurdestechnologieslaseret unechimiesol-gelpour intégrer des matériaux fonctionnels inorganiquessemi-conducteurs ou diélectriques, dans des procédés rapides, agiles, avec des résolutions spatiales aux échelles micro et nanométriques.

poussé. Cependant, ces procédés de fabrication sont très coûteux et rarement compatibles avec des substrats fragiles tels que le verre mince, les plastiques souples ou les pièces fabriquées par impression 3D. L'alternative consistant à déposer des couches minces à partir de matériaux en solution, par des procédés sol-gel, s'est rapidement développée ces dernières années. Ce procédé nécessite toutefois un traitement thermique à température élevée $\left(400-600^{\circ} \mathrm{C}\right)$ après le dépôt pour obtenir les propriétés semi-conductrices de la couche d'oxyde. Ce traitement est également incompatible avec les substrats minces et/ou flexibles, qui sont très recherchés dans de nombreuses applications de capteurs.

Les lasers sont maintenant largement utilisés dans l'industrie, dans différents contextes. Ils sont en particulier importants pour des problématiques de découpe ou soudage. Pour ces applications, c'est la possibilité de fournir une grande quantité d'énergie, localement, ce qui permet de définir précisément la zone irradiée, avec un contrôle temporel: le phénomène induit par laser est en général très rapide et 


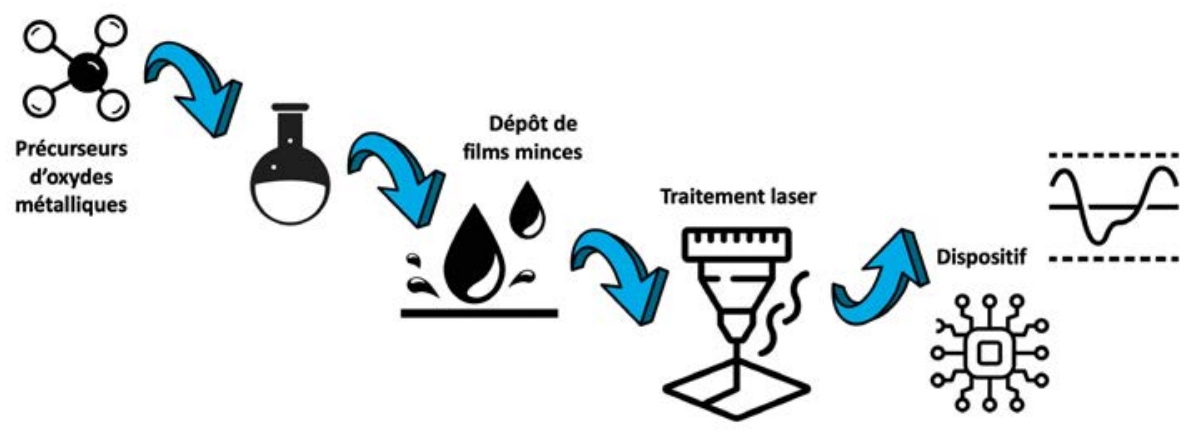

s'interrompt également rapidement quand la source est éteinte. Plus récemment, on a vu apparaître des applications nouvelles qui impliquent les lasers en fabrication additive.

Les exemples présentés dans cet article ont pour but de montrer l'intérêt de traitement laser pour fabriquer, par différentes approches physico-chimiques, des micro-nanostructures à base de matériaux inorganiques de type oxyde métallique. Le matériau est, à l'origine, sous une forme liquide, à l'état de molécules dissoutes dans un solvant et peut être facilement déposé sous forme de film mince. Le rôle de l'irradiation laser est de construire le matériau fonctionnel sur la base de briques élémentaires moléculaires, tout en définissant les zones traitées, ce qui se combine avec des perspectives de micro-nanofabrication.

\section{PRINCIPES DE BASE}

La problématique de base est de transférer avec un rendement maximal et à des échelles de temps réduites l'énergie lumineuse apportée par le faisceau laser en énergie utilisable pour initier efficacement d'interaction lumière-matière pour y parvenir, qui reposent sur :

Excitation directe. Dans ce cas particulier, on suppose 1 / que la matière première absorbe la lumière produite par le laser et 2/ qu'à partir des états excités produits par l'absorption de cette lumière sont produites des espèces chimiques qui permettent d'initier la réaction à la base de la fabrication du matériau. Cette voie est intéressante car il n'est pas nécessaire d'ajouter d'additifs à la formulation pour la rendre photosensible. En corolaire de cette propriété, une telle approche suppose une absorption importante du matériau à la longueur d'onde d'émission du laser et des phénomènes physico-chimiques induits efficaces à partir des états excités mais cette approche est en générale limitée à un couple laser/ matériau mais pas facilement transposable à d'autres cas.

Effets photothermiques. Dans ce cas, l'objectif est de générer une augmentation locale de température qui doit permettre aux processus chimiques de conduire à la mise en forme du matériau. Suivant la nature du matériau et de la source laser utilisée, il peut être nécessaire d'impliquer des photoabsorbeurs qui sont des molécules ou nanoparticules qui jouent le rôle de générateur de chaleur activés par l'irradiation.

Un deuxième challenge important dans les problématiques de micro-nanofabrication consiste à gérer le confinement de l'interaction lumière-matière aux échelles voulues et induire cette transformation à
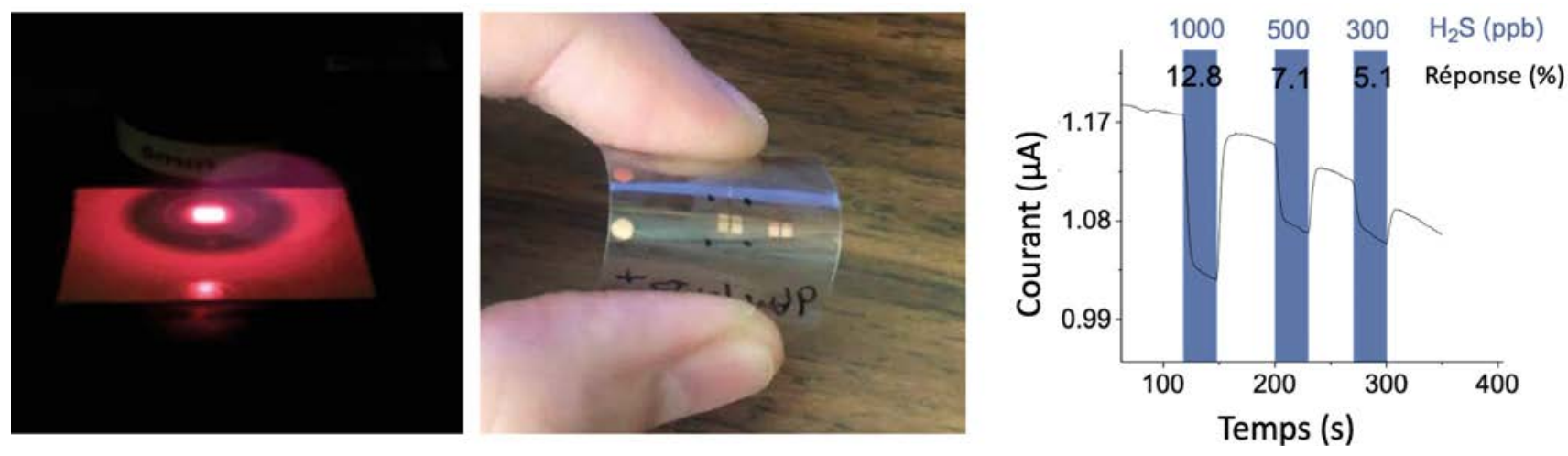
cette échelle, avec le minimum de pertes possibles en résolution.

La suite de cet article présente quelques exemples de réalisations récentes développées dans notre équipe. Ces exemples mettent en jeu des matériaux inorganiques (oxydes de métaux de transition). Les sources laser utilisées couvrent une large plage de longueur d'onde, allant des UV-profonds (DeepUV, $193 \mathrm{~nm}$ ) au proche infrarouge (NIR, $808 \mathrm{~nm}$ ). Les voies de traitement sont résumées, à l'échelle moléculaire dans l'encart 1.

\section{COUCHES DIÉLECTRIQUES ET SEMI-CONDUCTRICES D'OXYDES MÉTALLIQUES PRÉPARÉES PAR TRAITEMENT LASER UV-PROFOND À TEMPÉRATURE AMBIANTE}

Les niveaux d'énergie des photons dans l'UV profond (193 nm) correspondent à la gamme d'énergie nécessaire pour exciter directement un grand nombre de matériaux. En utilisant des fluences intermédiaires pour éviter les régimes d'ablation laser, il est possible d'utiliser cette interaction lumière matière pour réticuler des matériaux sol-gel. C'est en particulier très intéressant d'appliquer ce principe à la réticulation de matériaux sol-gel à base de métaux de transition (Ti, Zr, Hf, Zn...). Sous certaines conditions d'irradiation, cela permet de former directement les oxydes correspondants, sous forme de couche mince, à température ambiante, en quelques secondes d'irradiation. Ces phénomènes physico-chimiques peuvent être étudiés par différentes techniques d'analyse (ellipsométrie, FTIR, Raman, XPS, DRX). [1]

Ces travaux trouvent des applications intéressantes pour l'intégration de couches d'oxydes métalliques sur des substrats ne supportant pas $\bullet \bullet$

\section{LES DEUX VOIES DE FORMATION D'OXYDE MÉTALLIQUE PAR TRAITEMENT LASER DE COUCHES MINCES SOL-GEL}

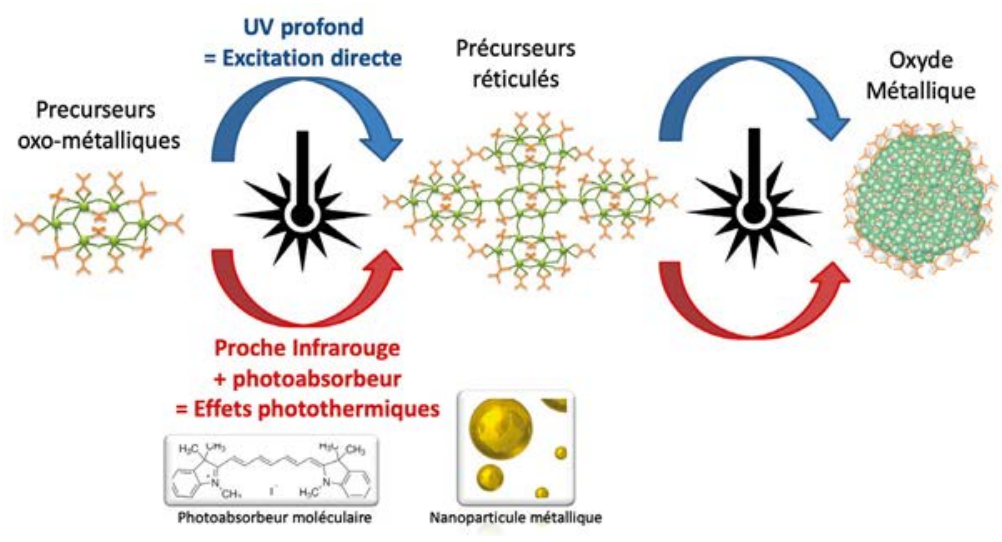

Résumé schématique des deux voies de traitement des précurseurs oxométalliques par irradiation laser UV profond ou proche infrarouge. Dans le premier cas, il est possible de générer une excitation directe des précurseurs qui conduisent par des réactions de condensation à une matrice de précurseurs réticulée, puis à l'oxyde métallique. Dans le deuxième cas, des photoabsorbeurs moléculaires ou des nanoparticules métalliques jouent le rôle de photoabsorbeurs pour provoquer la transformation du matériau vers l'oxyde aux propriétés semi-conductrices ou diélectriques suivant la nature du métal.

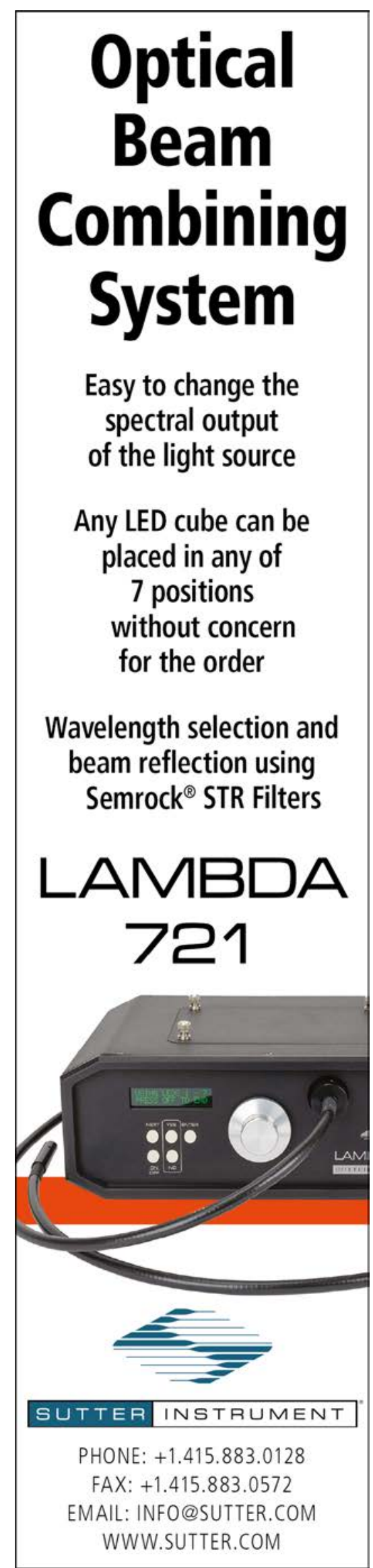




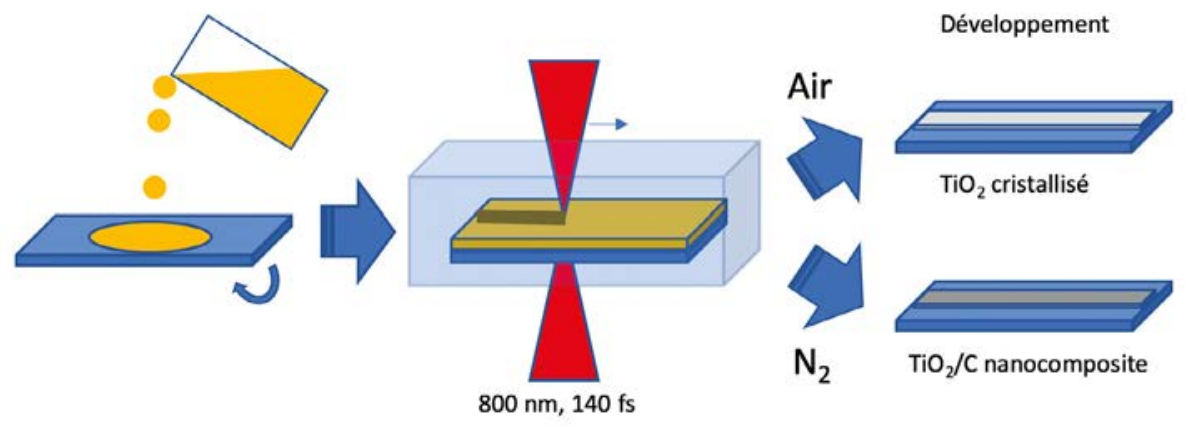

de traitement thermique, tels que les plastiques usuels. Récemment, nous avons montré l'intérêt de cette approche pour intégrer une couche mince transparente à haut indice dans des capteurs sur fibre optique polymère. [2] La forte absorption des matériaux dans ces gammes de longueur d'onde donne à cette approche un intérêt tout particulièrement pour l'élaboration de films minces diélectriques ou semi-conducteurs. En revanche, pour la même raison, il n'est pas possible d'envisager de traiter des couches épaisses de matériau ou du matériau massif. Cette approche peut en outre être facilement couplée à une micro ou nanostructuration par écriture laser directe ou en utilisant des outils de photolithographie classiques, ce qui en fait une technologie de choix parmi les autres approches de micro-nanostructuration d'oxydes métalliques préparés par voie liquide. [3]

\section{EFFETS PHOTOTHERMIQUES DANS LE PROCHE INFRA-ROUGE GÉNÉRÉS AVEC DES PHOTOABSORBEURS MOLÉCULAIRES}

Le traitement par laser UV-profond sur ce type de couches minces sol-gel est très pertinent pour adresser des problématiques de résolution jusqu’à l'échelle nanométrique en se basant sur le fait que la résolution spatiale prévue par la limite de diffraction est diminuée à faible longueur d'onde. Toutefois, il a été observé généralement que la conversion des précurseurs en oxyde métallique n'est pas complète. La conversion complète
Figure 3. Écriture par laser femtoseconde de $\mathrm{TiO}_{2}$ et $\mathrm{TiO}_{2} / \mathrm{C}$ à partir de films minces d'oxoclusters de Ti.

peut alors être obtenue par une irradiation dans le domaine du proche infrarouge.

Toutefois, la couche mince sol-gel à traiter est en général transparente dans la gamme de longueur d'onde infrarouge. Il est donc nécessaire d'utiliser un photoabsorbeur. Les cycles d'absorption/désexcitation non-radiative des molécules de photoabsorbeurs permettent ainsi d'atteindre localement les températures nécessaires pour convertir la couche déposée en oxyde qui suppose d'atteindre localement des températures de l'ordre de $300^{\circ}$ à $600^{\circ} \mathrm{C}$.

Figure 4. Vue schématique de l'approche thermoplasmonique pour la mise en œuvre d'un nanocomposite InZnO/Au NP impliquant une irradiation laser à $808 \mathrm{~nm}$. À droite, photographie d'un échantillon, la coloration est liée à la résonance plasmonique des nanoparticules d'or dans la couche InZnO.
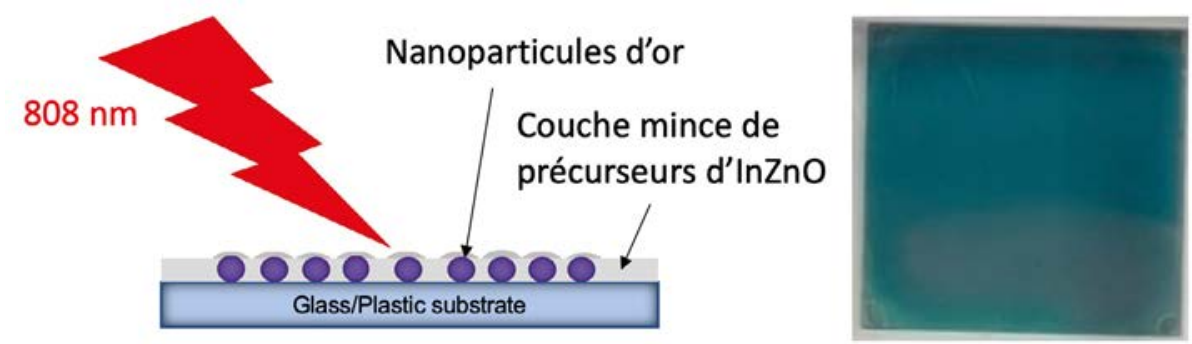

Des résultats montrent la possibilité de préparer des films minces (100 nm) de $\mathrm{ZnO}$ ou InZnO, sur des substrats plastiques ou même des textiles. L'objectif visé est ici de pouvoir intégrer des microstructures d'oxydes métalliques sur des substrats souples pour des applications en électronique flexible. Ces résultats ont été obtenus en utilisant des lasers continus NIR pour développer des capteurs de gaz qui peuvent être intégrés sur des plastiques car nous avons montré que la production de chaleur reste confinée dans le film et ne détériore pas le substrat. L'intérêt de la longueur d'onde NIR (808 $\mathrm{nm}$ dans ce cas) est d'être très peu absorbée par des plastiques usuels de type polycarbonate ou polypropylène. [4]

Ces approches peuvent être développées sur la base d'irradiations laser continues ou pulsées. Les régimes photothermiques sont différents en fonction de la source. Le recours à des irradiations laser courtes (femtoseconde), permet d'induire des transformations de la matière avec un contrôle fin. Dans le cas de précurseurs de $\mathrm{TiO}_{2}$, par excitation d'un photoabsorbeur, il est possible d'induire localement un échauffement très important qui permet de cristalliser le matériau pour obtenir du rutile microstructuré. Un aspect très intéressant repose sur la possibilité de mener ces réactions sous atmosphère inerte, ce qui conduit à une graphitisation des produits carbonés, et in fine à un nanocomposite $\mathrm{C} / \mathrm{TiO}_{2}$ qui a montré des propriétés conductrices qui peuvent être mises à profit pour élaborer des microcapteurs de pression [5]. 


\section{EFFETS THERMOPLASMONIQUES}

D'autres options sont possibles pour générer des effets photothermiques. Les nanoparticules métalliques sont connues pour leur effet plasmonique. L'effet plasmonique consiste en une vibration collective du nuage d'électrons libres dans un métal sous l'effet d'un champ électromagnétique. Cette vibration se traduit par un échauffement local du matériau, parfois très élevé pour certaines longueurs d'onde de la source d'excitation correspondant aux conditions de résonance du matériau. Cet effet bien connu est utilisé en photothérapie par exemple mais pas à notre connaissance pour initier des réactions de condensation de précurseurs sol-gel.

Dans ce contexte, nous avons proposé un nouveau procédé de fabrication simple et efficace dont l'originalité réside dans l'insertion de nanoparticules d'or à l'intérieur du film semi-conducteur d'oxyde d'indium/zinc (IZO).[6] Les nanoparticules d'or jouent un double rôle. D'une part, elles induisent, par effet plasmonique, un chauffage local sous l'effet d'un rayonnement infrarouge (laser), ce qui permet d'obtenir les propriétés semi-conductrices requises de la couche d'IZO sans affecter le substrat. Une monocouche de nanoparticules métalliques déposée à l'interface entre le substrat et le matériau à traiter est en effet suffisante pour obtenir les températures requises pour l'apparition des propriétés intéressantes. Par comparaison avec des échantillons préparés par voie thermique conventionnelle, nous avons pu montrer que le résultat sous irradiation était équivalent à celui obtenu à une température de $300^{\circ} \mathrm{C}$. En plus de réaliser ce rôle de photoabsorbeur crucial pour mettre en forme le matériau, les nanoparticules emprisonnées dans la matrice semi-conductrice jouent un deuxième rôle : elles confèrent à la couche mince des propriétés photorésistives (la résistivité évolue sous l'effet d'une illumination lumineuse) qui permettent la photodétection dans une large gamme de longueurs d'onde, de l'UV au proche infrarouge ( $365 \mathrm{~nm}$ à $780 \mathrm{~nm}$ ). Ce procédé permet ainsi d'intégrer un photodétecteur sur un film plastique souple en quelques étapes et avec des moyens très simples. Ce concept, pour lequel un brevet a été déposé, ouvre la voie à de nouveaux dispositifs microélectroniques tels que les capteurs chimiques, les détecteurs de mouvement, la surveillance du pouls et autres objets connectés.

\section{CONCLUSION}

Le couplage de techniques de traitement laser avec les approches de chimie solgel permettent de réaliser l'intégration de matériaux inorganiques aux propriétés semi-conductrices ou diélectriques de façon simple, adaptable et compatible avec des substrats fragiles car le traitement par laser permet de conserver un procédé à température ambiante. Ces travaux ont déjà montré un intérêt important dans le domaine des capteurs : chimiques, gaz, photodétecteurs, pression, déformation, etc... en tirant parti de la possibilité d'une intégration sur un grand nombre de substrats de natures différentes.
RÉFÉRENCES

[1] F. Stehlin et al., Journal of Materials Chemistry C 2, 277 (2014)

[2] N. Cennamo et al., ACS Applied Nano Materials 4, 10902-10910 (2021)

[3] C. C. Yeh et al., Advanced Materials 30, 24 (2018)

[4] P.-Y. Chang et al., ACS Applied Materials \& Interfaces 12, 24984 (2020)

[5] S. Y. Yu et al., Advanced Materials 30, 8 (2018)

[6] C.-F. Lin et al., Advanced Optical Materials 9, 2100045 (2021)

\section{Ultra-Short Pulse} Measurement \& Diagnostics

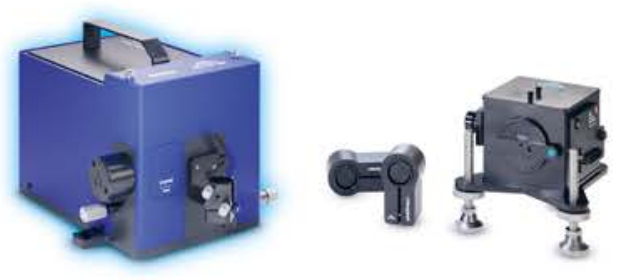

- New Autocorrelator

- Microscopy Autocorrelator

- Spectrometer

- SPIDER

- FROG

Come and visit us! 